\title{
Joint Reduction of PMEPR and Sidelobe in Multicarrier Radar Signals using $\mathrm{ZC}$ and SC Method
}

\author{
C.G. RAGHAVENDRA ${ }^{1 *}$, N. HARSHA ${ }^{1}$, MOHAN KUMAR $\mathrm{S}^{2}$ and PRASHANTHA H.S ${ }^{3}$ \\ ${ }^{1}$ Department of Electronics \& Communication Engineering, MSRIT, Bangalore, INDIA \\ ${ }^{2}$ Department of Computer Science and Engineering, MSRIT, Bangalore, INDIA \\ ${ }^{3}$ Department of Electronics \& Communication Engineering, NMIT, Bangalore, INDIA \\ *cgraagu@msrit.edu
}

\begin{abstract}
Employing the multitone waveforms for radar applications has attracted many authors in recent days. Several researchers have investigated the use of multitone waveform and challenges associated while implementation. The serious drawback of using multicarrier waveforms is the high Peak to Mean Envelope Power Ratio (PMEPR) values recorded and large sidelobes. To overcome PMEPR and sidelobe drawbacks a novel method is suggested for reducing sidelobe levels. The projected method applies zad-off chu phase sequence for multicarrier complementary phase coded (MCPC) signals. The comparison of integrated sidelobe ratio and peak sidelobe is obtained. Autocorrelations and complex envelope of the MCPC signal is obtained from the above methods are analyzed with extensive simulations. It is also possible to reduce the PMEPR and sidelobe simultaneously by using secondary user method. The suggested technique results with reduction in sidelobe and PMEPR levels.
\end{abstract}

Key-Words: - OFDM, MCPC, Zadoff-Chu sequence, Constellation, Primary User, Secondary User.

Received: September 7, 2019. Revised: January 8, 2020. Accepted: January 26, 2020. Published: February 5, 2020.

\section{Introduction}

In current day scenario there is a huge surge in demand for more information and the rapid growth of multimedia wireless applications, all points towards the need of a novel technology which aide very high speed data transmission rates. Similarly in the field of radar communications also there is a dire need for such new technique which balances the contrarian relationship between range and resolution detection capabilities. The range (or delay) resolution of a radar structure is inversely relative to the bandwidth of the signal transmitted. Therefore, the best evident way to enhance the range and resolution is to curtail the pulse width, because the pulse duration of each bit is inversely proportional to bandwidth [1]. On the other hand, for the better detection capacity of radar, the system must transmit with greater signal energy, and this can be attained by smearing long duration pulses, because the radar transmitting systems are operated close to their peak power limitations. One suggested method is to follow the pulse compression method to counterbalance the differences between the resolution and range of the radar signal.

There may be a huge thirst of bandwidth if single carrier methods are used for radar signal communication. But, taking the advantage of multitone or multicarrier methods, i.e., Orthogonal Frequency Division Multiplexing (OFDM) for the transmission of the radar signal may help to reduce the bandwidth requirements [2]. OFDM is a method of multiplexing the modulated signals. Multiplexing in general represents combing the subcarriers signals, which is formed by various sources. In multicarrier signal the multiplexing is applied to independent (or subcarrier) signals, however, these subcarrier signals are a sub-set of the complex (or main) signal. In OFDM the signal is divided into subcarriers, modulated by data (or phase) and later it is recombined to obtain the final OFDM signal. The concept of OFDM can be applied in radar for detecting the target.

In 2000 Levanon [3] coined a concept called as multicarrier or multitone complementary phase coded (MCPC) radar signal which was developed by the use of digital phase coded method. The Peak to Mean Envelope Power Ratio (PMEPR) in the MCPC signal is supposed to be less. In the conventional method of MCPC signal it was observed, if an attempt is made to lessen the PMEPR value, the sidelobe levels increases and vice versa, this may result into the wastage of energy due to more sidelobes. This will cause the weaker target echoed signal to hide. In addition, saturation of power amplifiers should be avoided along with the sidelobes which may reduce the efficiency.

Number of authors has explored and conducted several experiments to curb the influence of PMEPR on multicarrier schemes and significant emphasis is focused towards applying for data transmission 
systems, following are the methods followed such as improved tone reservation scheme with fast convergence [4], adaptive tone reservation scheme [5], SCR-Based tone reservation [6] and Iterative Re-Weighted [7]. Several attempts were made by the authors to weaken PMEPR levels in multitone signals for radar operations. Phase modulation technique is used in [8], PMEPR condensed using ILS technique in [9], GA method has been used [10] and Random Phase Algorithm is used in [11]. In reducing the sidelobes few authors has attempted using constellation adjustment method in [12]. A sidelobe level of $35 \mathrm{~dB}$ is reduced using genetic algorithm and reported in [13] but computational complexity is high. All the above cited literatures will try to reduce either PMPER or sidelobe separately. However, few authors have focused for jointly addressing the two problems which is reported in the literatures [14-16], but focus is on non-radar signal issues. In the article [17] ZadoffChu (ZC) phase method is applied for radar application and in this paper same is extended with SU method for reducing autocorrelation sidelobe level and maintaining low value of PMEPR. This paper is organized as follows. Section 2 summarizes the multitone signal structure. MCPC implementation based on $\mathrm{ZC}$ phase sequence is presented in Section 3. MCPC implementation based on $\mathrm{SC}$ technique is mentioned in section 4 with extensive simulation results. In section 5 conclusions are drawn which highlights the ability of proposed technique.

\section{The Multitone Signal Structure}

The multifrequency phase coded radar signal is based on the theory of OFDM scheme. The MCPC signal employs $\mathrm{P}$ number of carriers which is composed of $\mathrm{P}$ subcarriers transmitted all in parallel, which are parted in frequency by inverse of each duration of $t_{c}$ bit. This concept of splitting the frequency yields OFDM notion, which is well known in the field of communication. In the OFDM every carrier is phase modulated with $\mathrm{P}$ number of bits and each bit will be of $t_{c}$ duration and these phases are developed with polyphase codes, namely P3 and P4. A schematic block diagram for developing MCPC signal which is based on polyphase P4 codes is shown in Fig. 1.

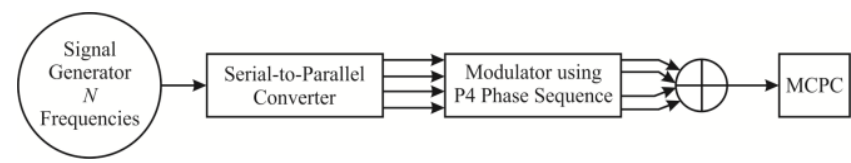

Fig.1. Block diagram of MCPC-OFDM signal generator

\subsection{Mathematical Expression for MCPC Signal}

The time domain representation of MCPC-OFDM signal is developed with the following equations

$$
x(t)=\left\{\begin{array}{cc}
\sum_{q=1}^{P} W_{a} \exp \left\{j\left[2 \pi t f_{p}(((p+1) / 2)-q)+\theta_{q}\right]\right\} \\
\sum_{p=1}^{P} u_{q, p}\left[t-(p-1) t_{p}\right], \quad 0 \leq t \leq t_{p} \\
0, \quad \text { elsewhere }
\end{array}\right.
$$

Where $u_{q, p}(t)=\left\{\begin{aligned} \exp \left(j \phi_{q, p}\right), & 0 \leq t \leq t_{p} \\ 0, & \text { elsewhere }\end{aligned}\right.$

where $\phi_{\mathrm{q}, \mathrm{p}}$ indicates the $\mathrm{p}^{\text {th }}$ phase of the $\mathrm{q}^{\text {th }}$ row series. Here $\theta_{\mathrm{q}}$ is assumed as random phase shift reckoned to every sub-carrier by the transmitting signal and $W_{a}$ implies the amplitude scaled for the $\mathrm{n}^{\text {th }}$ sub-carrier. Equation 3 is polyphase code of type P4 used to develop the phase sequence and mentioned as

$\phi_{p}=\frac{\pi(p-1)^{2}}{P}-\pi(p-1) \quad p=1,2,3 \ldots \ldots . P$

By assuming $\mathrm{P}=5$ and by repeatedly differing the values of $P$ from $1,2, \ldots, 5$. Then several phase values are attained and it is enumerated in Table 1.

Table 1: Periodically shifted complimentary codes with length of $\mathrm{P}=5$

\begin{tabular}{|c|l|l|l|l|l|}
\hline $\begin{array}{l}\text { Sequence } \\
\text { No }\end{array}$ & \multicolumn{4}{|l|}{ P4 Phase elements } \\
\hline 1 & $0^{\circ}$ & $-144^{\circ}$ & $-216^{\circ}$ & $-216^{\circ}$ & $-144^{\circ}$ \\
\hline 2 & $-144^{\circ}$ & $-216^{\circ}$ & $-216^{\circ}$ & $-144^{\circ}$ & $0^{\circ}$ \\
\hline 3 & $-216^{\circ}$ & $-216^{\circ}$ & $-144^{\circ}$ & $0^{\circ}$ & $-144^{\circ}$ \\
\hline 4 & $-216^{\circ}$ & $-144^{\circ}$ & $0^{\circ}$ & $-144^{\circ}$ & $-216^{\circ}$ \\
\hline 5 & $-144^{\circ}$ & $0^{\circ}$ & $-144^{\circ}$ & $-216^{\circ}$ & $-216^{\circ}$ \\
\hline
\end{tabular}

By shifting cyclically, the elements of first row mentioned in Table 1 for $\mathrm{P}$ times, results in generation of $\mathrm{P}$ different complementary sets of sequences. At the same time applying all the phase values simultaneously to the multiple sub-carriers, the coded signals are attained. The phase modulation follows the frequency ordering of $\left(-2 f_{p}\right.$, $\left.-f_{p}, 0, f_{p}, 2 f_{p}\right)$ along the subcarriers. The $P$ subcarriers used with a frequency gap of $f_{p}=1 / t_{p}$, where $t_{p}$ is the interval between the modulated phase elements. By performing the time domain addition of these modulated signal gives $\mathrm{P} \times \mathrm{P} \mathrm{MCPC}$ signals. From equations 1, 2 and 3 it is possible to generate the MCPC signal structure with the subcarrier combinations of $7 \times 7,9 \times 9,11 \times 11$ and so on. 


\subsection{Simulation Results of P4 based MCPC Signal}

The order of the phase combination in MCPC signal represents the phase sequence being employed to modulate a particular subcarrier. To develop the MCPC signal with [ [ $\left.\begin{array}{lllll}3 & 5 & 2 & 1 & 4\end{array}\right]$ order, the phase sequence of 3rd row mentioned in Table 1 is phase modulated with the first subcarrier, $5^{\text {th }}$ row is phase modulated with second subcarrier, $2^{\text {nd }}$ sequence is phase modulated with the $3^{\text {rd }}$ subcarrier and so on.

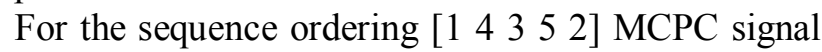
is developed as shown in the Fig. 2. Fig. 3 shows the corresponding autocorrelation plot. Similar

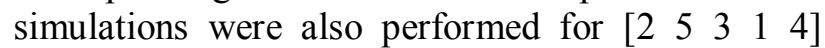
ordering and the same has been included below.

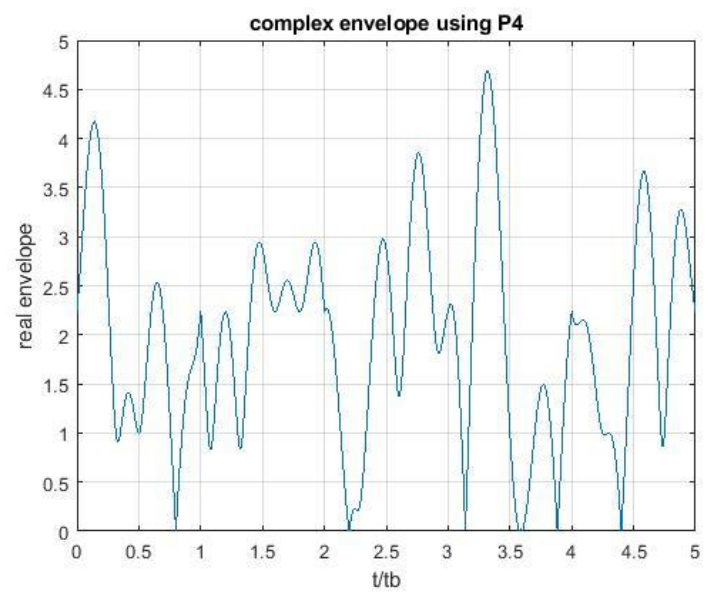

Fig. 2. Conventional MCPC signal for $5 \times 5 \mathrm{MCPC}$ $\left[\begin{array}{lllll}1 & 4 & 3 & 2 & 5\end{array}\right]$

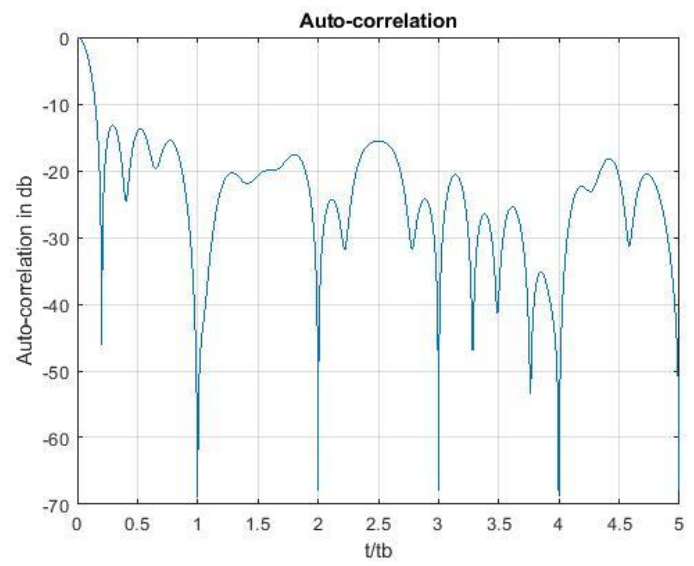

Fig. 3. Autocorrelation plot for $5 \times 5$ MCPC $\left[\begin{array}{llll}1 & 4 & 3 & 2\end{array}\right.$ 5]

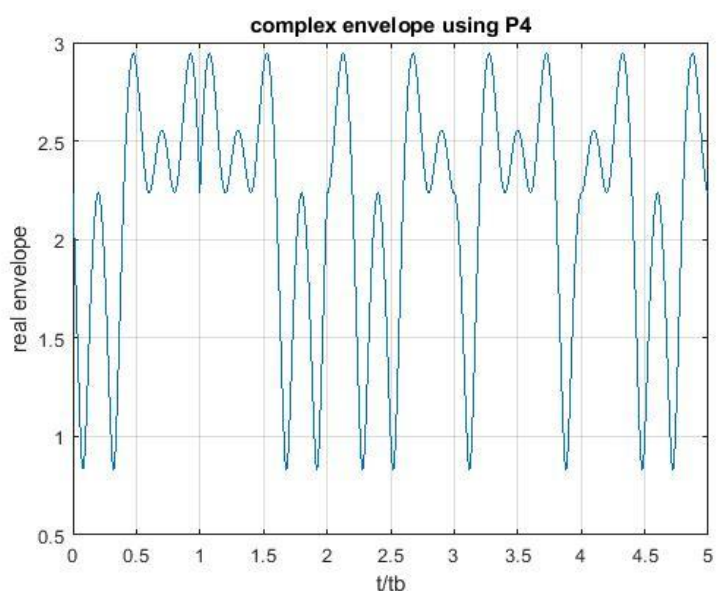

Fig. 4. Conventional MCPC for $5 \times 5$ MCPC [2 531 4]

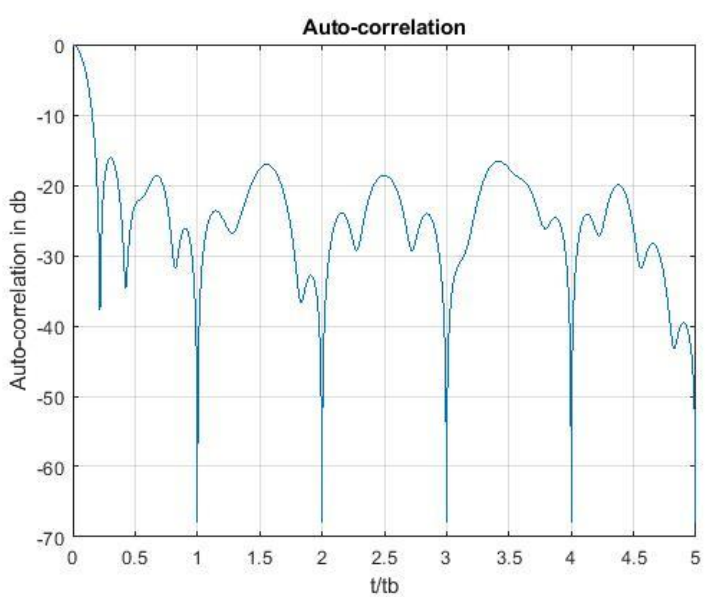

Fig. 5. Autocorrelation plot for $5 \times 5$ MCPC $\left[\begin{array}{llll}2 & 5 & 3 & 1\end{array}\right.$ 4]

Table 2. PMEPR and Sidelobe Levels of $5 \times 5$ MCPC Signal

\begin{tabular}{|l|l|l|}
\hline $\begin{array}{l}\text { MCPC for various } \\
\text { ordering with 5x5 }\end{array}$ & PMEPR & $\begin{array}{l}\text { Sidelobe in } \\
(\mathrm{dB})\end{array}$ \\
\hline 14352 & 4.3988 & -13.61 \\
\hline 25314 & 1.7338 & -12.4 \\
\hline
\end{tabular}

From Table 2, it is evident that the autocorrelation sidelobe level are $-12 \mathrm{~dB}$ and $-13 \mathrm{~dB}$

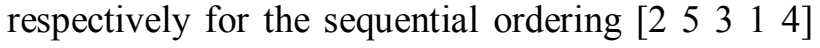
and $\left[\begin{array}{lllll}1 & 4 & 3 & 5 & 2\end{array}\right]$. Thus it proves our claim that there is further scope for improvement in dwindling the sidelobe level. To overcome this drawback, in this paper we suggest a new method for developing MCPC signal which is based on ZC phase values.

\subsection{Ambiguity Function of MCPC Signal}

The output of the matched filter to the signal from the target which is identical to the value of the ambiguity function of MCPC signal at $(\mathrm{t}, \mathrm{v})=(0,0)$. However, this function is used to calculate the 
Doppler resolution and range for a particular radar waveform. The ambiguity function can be expressed as shown below

$$
|\chi(\tau, v)|=\left|\int_{-\infty}^{\infty} x(t) x^{*}(t+\tau) \exp (j 2 \pi v t) d t\right|
$$

where $x$ is the absolute complex envelope of the signal. The positive $v$ represents the target reaching towards the radar system. Positive $\tau$ represents the target moving away from the radar. The ambiguity function is a key concept for analyzing or investigating radar signals. Fig. 6 shows the ambiguity function of MCPC signal for the sequence ordering [ $\left[\begin{array}{lllll}3 & 5 & 2 & 1 & 4\end{array}\right]$.

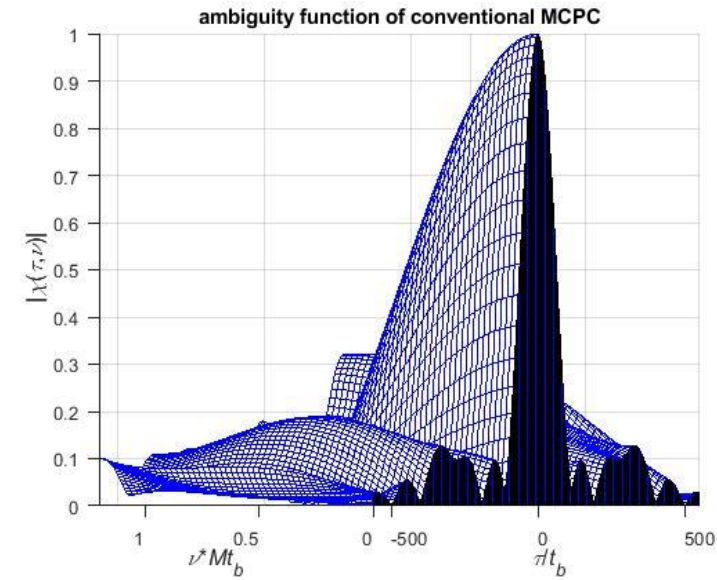

Fig. 6. Ambiguity function of $5 \times 5 \mathrm{MCPC}$ pulse for sequence order [ [ $\left.\begin{array}{lllll}3 & 5 & 2 & 1 & 4\end{array}\right]$

\section{ZC Phase Sequence MCPC based}

The projected ZC method will perform Hadamard product of the signal block with the equivalent $\mathrm{ZC}$ phase value of the $\mathrm{ZC}$ structure which is attained from the ZC matrix. Every factor in the ZC sequence characterizes complex number. Each ZC complex value will have a phase rotation which alters the corresponding phase of the signal element. This forms the formation of new version of $\mathrm{ZC}$ based MCPC signal.

The $\mathrm{ZC}$ root sequence of $\mathrm{n}^{\text {th }}$ complex value is shown in the below equation

$Z_{r}=\left\lfloor Z_{1 r}, Z_{2 r}, \ldots \ldots . ., Z_{N_{Z C_{r}}}\right\rfloor$

can be obtained by the following formula

$Z_{n}^{r}=e^{\left(\frac{-j \pi n(n+1)}{N_{Z c}}\right)}$

where NZC is assumed as the length of the ZC sequence. $1 \leq \mathrm{n} \leq \mathrm{NZC}, 1<\mathrm{g}<\mathrm{NZC}$, gcd (NZC, g) $=1$ and $\mathrm{q} \in \mathrm{Z}$. By exploiting the special property of $\mathrm{ZC}$ sequence we obtain the $\mathrm{ZC}$ matrix $\mathrm{Z}$, which is given in equation 7 .

$$
\begin{aligned}
& Z=\left(\begin{array}{ccc}
Z_{11} & \ldots \ldots . & Z_{1 N z c} \\
\vdots & \ddots & \vdots \\
Z_{N z c 1} & \ldots \ldots . & Z N_{z c} N_{z c}
\end{array}\right)=\left[Z_{1}, Z_{2}, \ldots \ldots . . Z_{N C}\right] \\
& Z_{n}=\left[Z_{1 n}, Z_{2 n}, \ldots . . ., Z_{N Z c n}\right]^{T}=Z^{r} \\
& n=1,2, \ldots . . N_{Z c}
\end{aligned}
$$

where, $Z_{\mathrm{r}}$ is the $\mathrm{ZC}$ root sequence whereas $Z_{\mathrm{n}}$ is the $(n-1)^{\text {th }}$ which is a shifted form of the ZC root sequence. The $\mathrm{ZC}$ codes phase values are shown in the Table 3.

Table 3. Periodically shifted complimentary codes using ZC

\begin{tabular}{|l|l|l|l|l|l|}
\hline $\begin{array}{l}\text { Sequ } \\
\text { ence } \\
\text { No }\end{array}$ & \multicolumn{5}{|l|}{ ZC Phase values } \\
\hline 1 & 0 & 1.2566 & 1.2566 & 0 & $\begin{array}{l}-2.513 \\
3\end{array}$ \\
\hline 2 & 1.2566 & 1.2566 & 0 & $\begin{array}{l}-2.513 \\
3\end{array}$ & 0 \\
\hline 3 & 1.2566 & 0 & $\begin{array}{l}-2.513 \\
\text { (n) }\end{array}$ & 0 & 1.2566 \\
\hline 4 & 0 & $\begin{array}{l}-2.513 \\
3\end{array}$ & 0 & 1.2566 & 0 \\
\hline 5 & -2.513 & 1.2566 & 1.2566 & 1.2566 & 0 \\
\hline
\end{tabular}

Every subcarrier is modulated with ZC phase values which are mentioned in Table 3 . These modulated subcarriers are added continuously which leads to generation of MCPC signal based on ZC sequence as shown in Fig. 7.

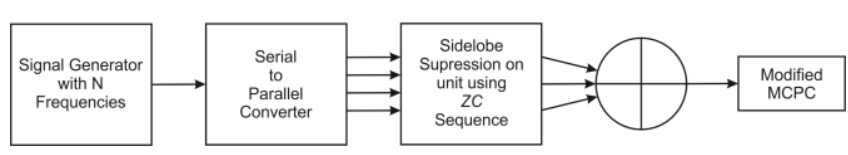

Fig. 7. Block diagram for developing $\mathrm{ZC}$ sequence based MCPC signal

In Table 4, comparison of MCPC signal which is based on $\mathrm{P} 4$ and $\mathrm{ZC}$ sequence is shown. For the various sequence ordering peak sidelobe level and peak of $1^{\text {st }}$ sidelobe level are examined. Fig 8 to Fig 11 shows the corresponding MCPC plots of autocorrelation function and real envelope for the sequence ordering [llllll $\left.\begin{array}{lllll}1 & 4 & 3 & 5 & 2\end{array}\right]$ and [ [ $\left.\begin{array}{lllll}2 & 5 & 3 & 1 & 4\end{array}\right]$ respectively. 
Table 4. Various Sidelobe of MCPC signal based on P4 with ZC Codes

\begin{tabular}{|c|c|c|c|c|c|}
\hline \multirow{2}{*}{ Sequence } & \multicolumn{3}{|c|}{ P4 based MCPC } & \multicolumn{2}{|c|}{$\begin{array}{l}\text { ZC based } \\
\text { MCPC }\end{array}$} \\
\hline & $\begin{array}{l}\text { PME } \\
\text { PR }\end{array}$ & $\begin{array}{l}1 \mathrm{st} \\
\mathrm{SLL}\end{array}$ & $\begin{array}{l}\text { Peak } \\
\text { SLL }\end{array}$ & $\begin{array}{l}1 \mathrm{st} \\
\text { SLL }\end{array}$ & $\begin{array}{l}\text { Peak } \\
\text { SLL }\end{array}$ \\
\hline 32514 & 2.9764 & -13.85 & -13.85 & -16.29 & -14.07 \\
\hline 53214 & 3.4821 & -19.16 & -14.92 & -22.71 & -15.30 \\
\hline 24351 & 2.9764 & -12.76 & -12.7 & -18.96 & -14.09 \\
\hline 13452 & 3.4821 & -12.32 & -12.32 & -16.31 & -12.34 \\
\hline 23514 & 2.9764 & -12.27 & -12.27 & -16.64 & -13.57 \\
\hline 14325 & 3.4821 & -16.31 & -12.26 & -22.67 & -15.06 \\
\hline 14253 & 1.7338 & -16.31 & -14.42 & -19.28 & -15.68 \\
\hline 24315 & 2.9764 & -13.72 & -12.62 & -16.78 & -13.71 \\
\hline 14352 & 4.3988 & -13.16 & -13.19 & -13.19 & -13.19 \\
\hline 25314 & 1.7338 & -16.16 & -15.16 & -19.28 & -15.72 \\
\hline
\end{tabular}

A PMEPR of 3.4821 is obtained for the sequence ordering [ [ $\left.\begin{array}{lllll}1 & 4 & 3 & 2 & 5\end{array}\right]$, both for $\mathrm{P} 4$ and $\mathrm{ZC}$ based sequences. For the MCPC signal based on ZC, $-22.67 \mathrm{~dB}$ of $1^{\text {st }}$ sidelobe level and peak sidelobe level of $-15.06 \mathrm{~dB}$ is obtained and 1st sidelobe level of $-16.31 \mathrm{~dB}$ and peak sidelobe level of $-12.26 \mathrm{~dB}$ is attained for MCPC based on P4 for the same sequence ordering. Correspondingly a PMEPR of 1.73 is obtained for P4 and ZC based MCPC signal for the sequence ordering $\left[\begin{array}{lllll}1 & 4 & 2 & 5 & 3\end{array}\right]$. The $1^{\text {st }}$ sidelobe level of $-19.28 \mathrm{~dB}$ and peak sidelobe level of $-15.68 \mathrm{~dB}$ is attained for MCPC based on $\mathrm{ZC}$ and the $1^{\text {st }}$ sidelobe level of $-16.31 \mathrm{~dB}$ and peak sidelobe level of $-14.42 \mathrm{~dB}$ is obtained for MCPC based on P4. It can be observed from the Table 4 for the sequence ordering [ $\left[\begin{array}{lllll}1 & 4 & 3 & 2 & 5\end{array}\right]$ an augment of nearly $3 \mathrm{~dB}$ of peak sidelobe and $6 \mathrm{~dB}$ in $1^{\text {st }}$ sidelobe for MCPC signal based on ZC obtained, when it is correlated to MCPC signal based on P4.

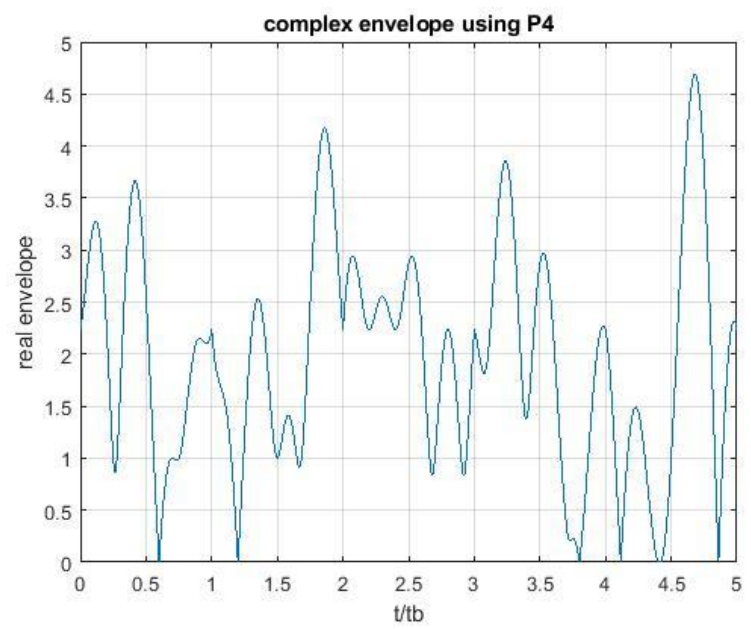

Fig. 8. Real envelope of 5x5 MCPC [ [ $\left.\begin{array}{llll}1 & 4 & 3 & 2\end{array}\right]$

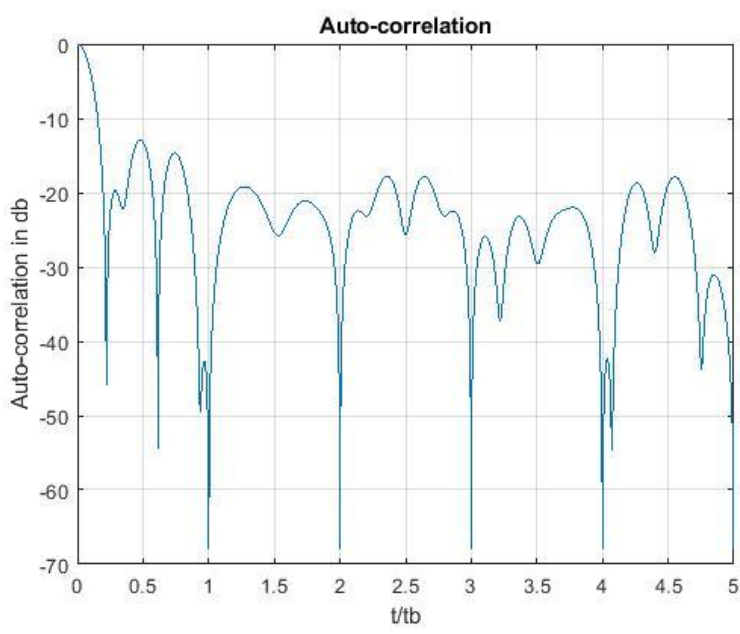

Fig. 9. Autocorrelation for 5x5 MCPC [ $\left[\begin{array}{lllll}1 & 4 & 3 & 2 & 5\end{array}\right]$

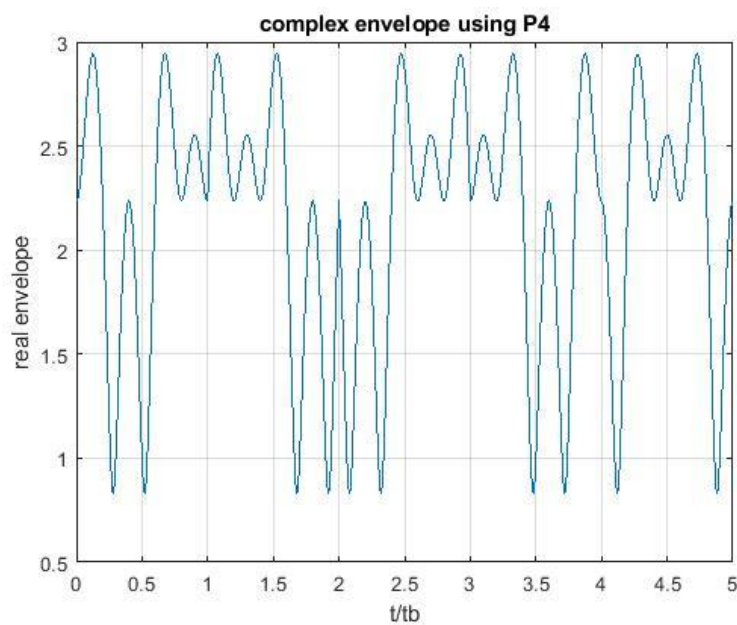

Fig. 10. Real envelope of $5 \times 5 \mathrm{MCPC}$ for the sequence order [ [ $\left.\begin{array}{lllll}2 & 5 & 3 & 1 & 4\end{array}\right]$

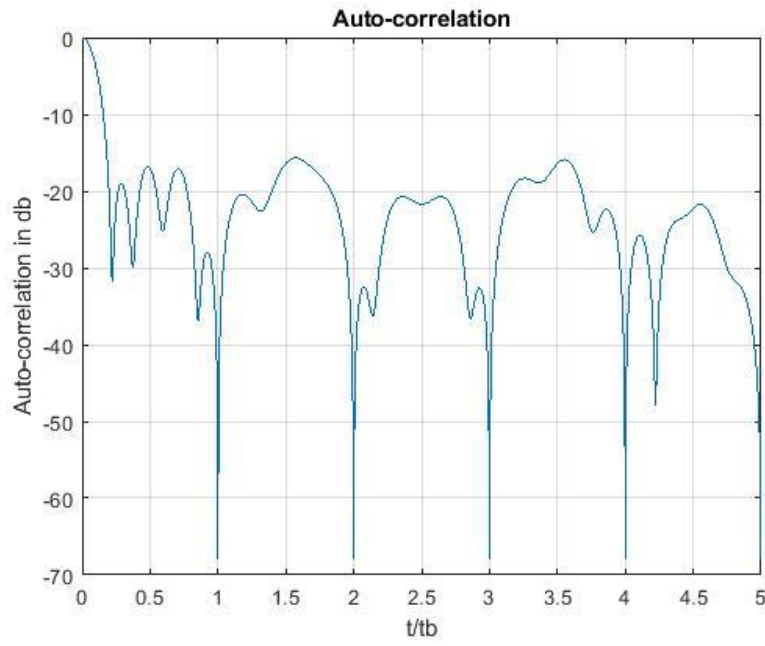

Fig. 11. Autocorrelation of $5 \times 5 \mathrm{MCPC}$ for the sequence order [ $\left[\begin{array}{lllll}2 & 5 & 3 & 1 & 4\end{array}\right]$ 


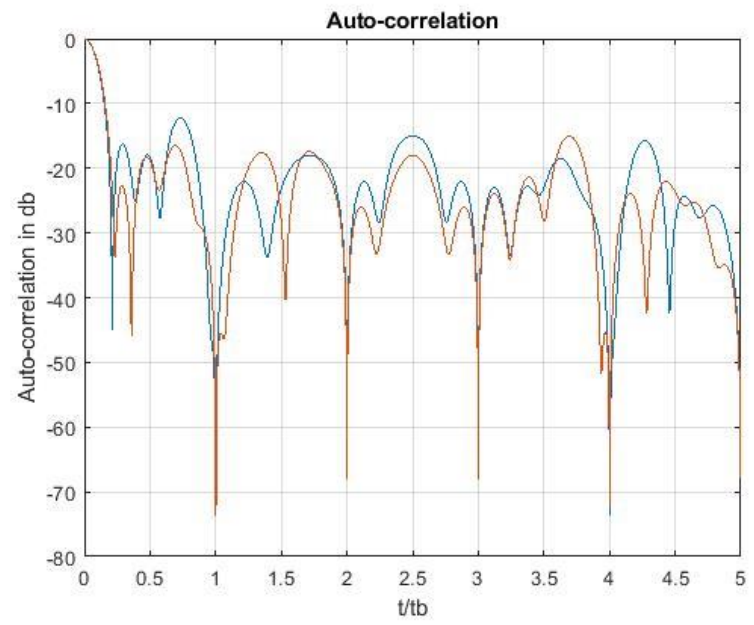

Fig. 12. Autocorrelation plots of MCPC using both P4 and ZC sequences

The Fig. 12 shows the comparison of sidelobe levels in MCPC using P4 and ZC sequences. The blue color waveform is obtained by using $\mathrm{P} 4$ sequence and red color waveform is obtained by using the $\mathrm{ZC}$ sequences. It can be observed from the autocorrelation function plot, an improvement in sidelobe level using ZC based MCPC signal when compared with $\mathrm{P} 4$ polyphase sequence based MCPC. In the Table 5, a comparison of integrated sidelobe level ratio (ISLR) for MCPC signal based on $\mathrm{P} 4$ and $\mathrm{ZC}$ is attained for various ordering. For [4 $\left.\begin{array}{llll}1 & 5 & 3 & 2\end{array}\right]$ ordering an improvement of $1.36 \mathrm{~dB}$ is obtained for MCPC based on ZC sequence method. Similarly Table 6 shows the comparison of peak sidelobe level ratio (PSLR) for MCPC based on P4 and ZC. For [ [ $\left.\begin{array}{lllll}4 & 1 & 5 & 3 & 2\end{array}\right]$ ordering an improvement of $2.38 \mathrm{~dB}$ is obtained for MCPC based on $\mathrm{ZC}$ sequence method.

Table 5. ISLR comparison of $\mathrm{P} 4$ and $\mathrm{ZC}$ sequences

\begin{tabular}{|c|c|c|}
\hline \multirow{2}{*}{ Sequence } & \multicolumn{2}{|l|}{$\begin{array}{l}\text { Integrated sidelobe level ratio (ISLR) of } \\
\text { MCPC }\end{array}$} \\
\cline { 2 - 3 } & Based on P4 & Based on ZC \\
\hline 41325 & 1.4523 & 1.3054 \\
\hline 42315 & 2.0750 & 1.2935 \\
\hline 13425 & 1.6484 & 1.4515 \\
\hline 41352 & 1.8043 & 1.3626 \\
\hline 13524 & 1.5031 & 1.2081 \\
\hline 31542 & 1.5988 & 1.4816 \\
\hline 52341 & 1.3869 & 1.2408 \\
\hline 41532 & 1.6996 & 1.3935 \\
\hline 13254 & 1.6465 & 1.6038 \\
\hline 42351 & 1.2576 & 1.1306 \\
\hline
\end{tabular}

Table 6. PSLR comparison of P4 and ZC sequences

\begin{tabular}{|l|c|c|}
\hline \multirow{2}{*}{ Sequence } & \multicolumn{2}{|c|}{$\begin{array}{l}\text { Peak sidelobe level ratio (PSLR) of } \\
\text { MCPC }\end{array}$} \\
\cline { 2 - 3 } & Based on P4 & Based on ZC \\
\hline 52341 & 0.2782 & 0.1811 \\
\hline 41352 & 0.2085 & 0.1891 \\
\hline 51432 & 0.2725 & 0.2527 \\
\hline 13254 & 0.2219 & 0.1823 \\
\hline 42351 & 0.2435 & 0.1823 \\
\hline 42315 & 0.2031 & 0.1933 \\
\hline 523441 & 0.2076 & 0.1711 \\
\hline 41325 & 0.1713 & 0.1462 \\
\hline 41532 & 0.2851 & 0.2810 \\
\hline 135224 & 0.2087 & 0.2041 \\
\hline
\end{tabular}

\section{MCPC Based on Signal Cancellation Method}

The fundamental concept in signal cancellation technique is to prolong the part of the constellation points on the Secondary User (SU) sub-carriers and inert the suitable cancellation symbols on Primary User (PU) subcarrier to produce the cancellation signal to decrease both PMEPR and sidelobe levels [13]. Here adding the cancellation symbols which cancels the part of the signal is considered at the transmitter. The signal cancellation (SC) method frames this problem as a quadratically constrained quadratic program to obtain optimal cancellation signal.

In the suggested $\mathrm{SC}$ approach, the constellation modification of the symbol Cs $=[\operatorname{Cs}(0), \operatorname{Cs}(1), \ldots$ $\mathrm{Cs}(\mathrm{N}-1)]$ is combined with the primary data symbol $\mathrm{X}$, where $\mathrm{Cs}(\mathrm{k})=0$ for $\mathrm{k} \notin \mathrm{R}$ on the SU subcarriers. Since we are not considering the SU subcarriers we concentrate only on the PU subcarriers. So, on the PU sub-carriers we add the signal cancellation (SC) symbol $\mathrm{Cp}=[\mathrm{Cp}(0), \mathrm{Cp}(1), \ldots, \mathrm{Cp}(\mathrm{N}-1)]$ to the primary data symbol $\mathrm{X}$. Where $\mathrm{Cp}(\mathrm{k})=0$ for $\mathrm{k} \in \mathrm{R}$. If we consider $\mathrm{C}=\mathrm{Cs}+\mathrm{Cp}$ as the total SC symbol of the $\mathrm{SC}$ technique, the following conditions must be satisfied

$C(k)= \begin{cases}C_{s}(k), & k \in R \\ C_{P}(k), & k \notin R,\end{cases}$

Here Cs is not considered, then the total SC symbol will be $\mathrm{C}=\mathrm{Cp}$ and the transmitted symbol $\hat{X}$ of the SC technique can be shown as

$\hat{X}=X+C_{p}=X+C$ 
The transmitted time domain signal $\hat{X}$ of the SC technique can be attained by the following equation

$$
\hat{X}=K X+K C_{s}+K C_{p}=K X+K C
$$

Hence the joint PMEPR reduction and sidelobe reduction issues can be framed as an optimization problem, i.e,

$$
\begin{array}{cl}
\min _{C} & \|K X+K C\|_{\infty} \\
\text { subject to } & \|X+C\|_{2} \leq \beta\left\|_{X}\right\|_{2} \\
& \|X+C\|_{2}^{2} \leq \varepsilon \\
& C(k) \in M, \text { for } k \in R
\end{array}
$$

where in the above constraints represents different objective functions in which $\beta$ represents the factor to control the maximum sidelobe power. By solving the above constraints which are the convex sets through the convex optimization tool, we obtain the optimal cancellation signal. But this involves computational complexity in solving the constraints. To address the quadratically constrained quadratic program (QCQP) problem, the solution is to generate the SC symbol which is based on the required constraints by considering one of the data symbol with opposite magnitude as the SC symbol (i.e., cancelling one of the subcarriers or one of the permutation of frequencies at the transmitter).

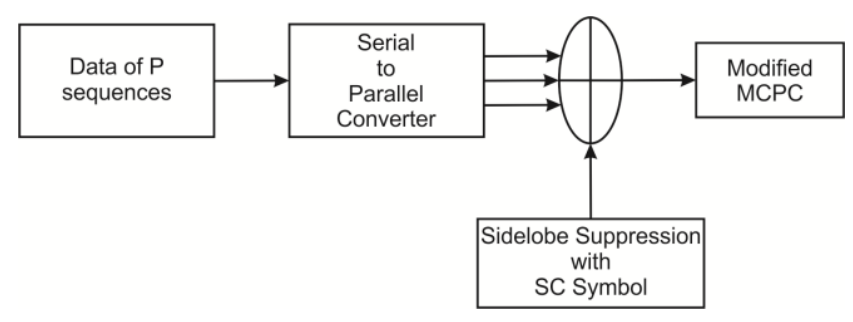

Fig. 13. Block diagram of MCPC using SC method

The above Fig. 13 shows the block diagram of developing MCPC signal based on SC symbol which suppress the sidelobe. This SC symbol is added with the modulated subcarriers to generate the modified MCPC signal with different phase showing better sidelobe levels when compared with conventional MCPC method which uses P4 sequence. The following section shows the simulation results of MCPC based on SC method. Fig. 14 and 15 shows the real and autocorrelation plot of MCPC signal developed based on SC symbol for [ $\left[\begin{array}{lllll}1 & 4 & 2 & 3 & 5\end{array}\right]$ ordering. Similarly Fig. 16 and 17 shows the real and autocorrelation plot of MCPC signal based on SC symbol for [ [ $\left.\begin{array}{lllll}4 & 5 & 2 & 1 & 3\end{array}\right]$ ordering.

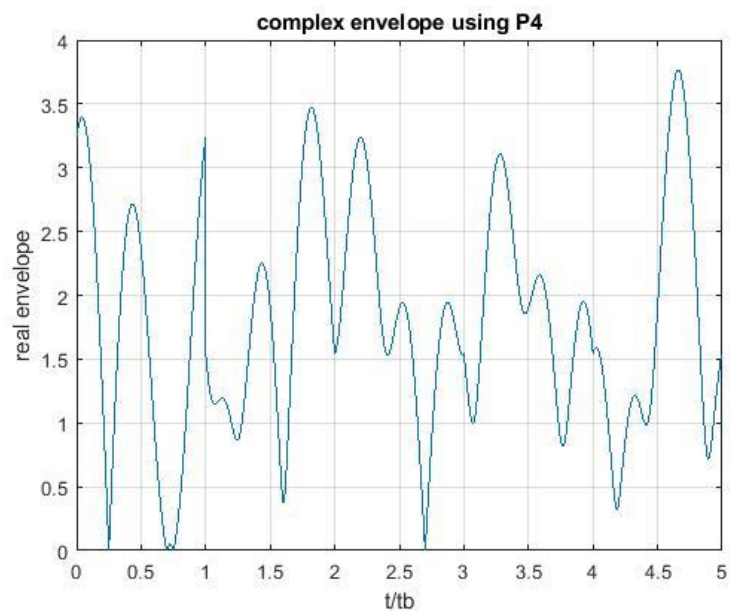

Fig. 14. Real plot of MCPC using SC for the sequence order [ $\left[\begin{array}{lllll}1 & 4 & 2 & 3 & 5\end{array}\right]$

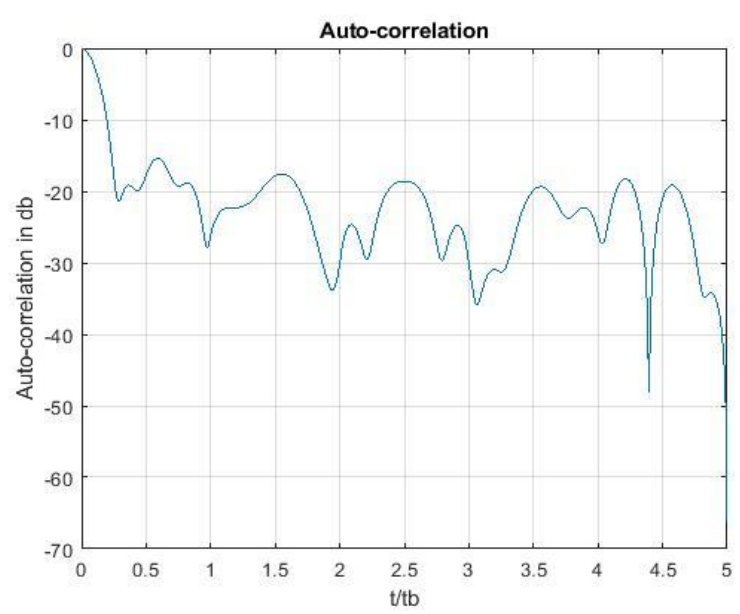

Fig. 15. Autocorrelation plot of MCPC using SC for the sequential order [ $\left[\begin{array}{lllll}1 & 4 & 2 & 3 & 5\end{array}\right]$

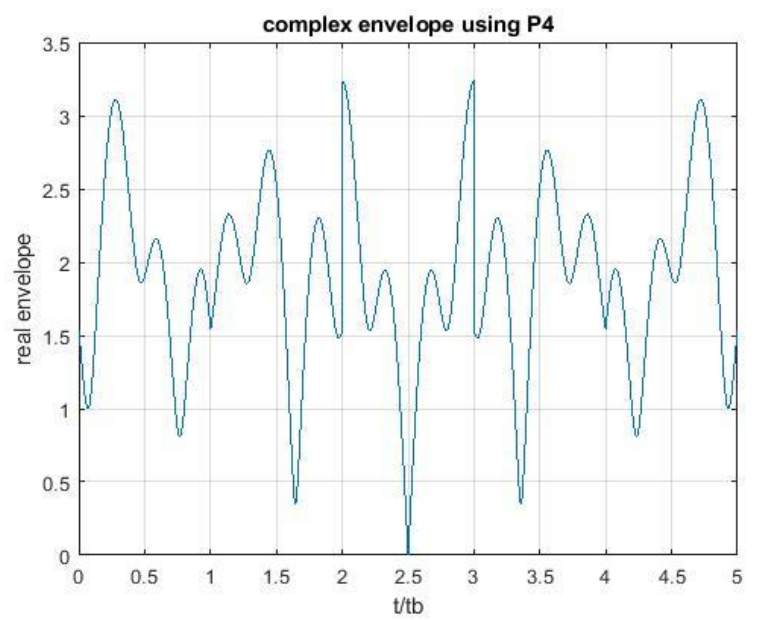

Fig. 16. Real plot of the MCPC using SC for the sequence order [ [ $\left.\begin{array}{lllll}4 & 5 & 2 & 1 & 3\end{array}\right]$ 


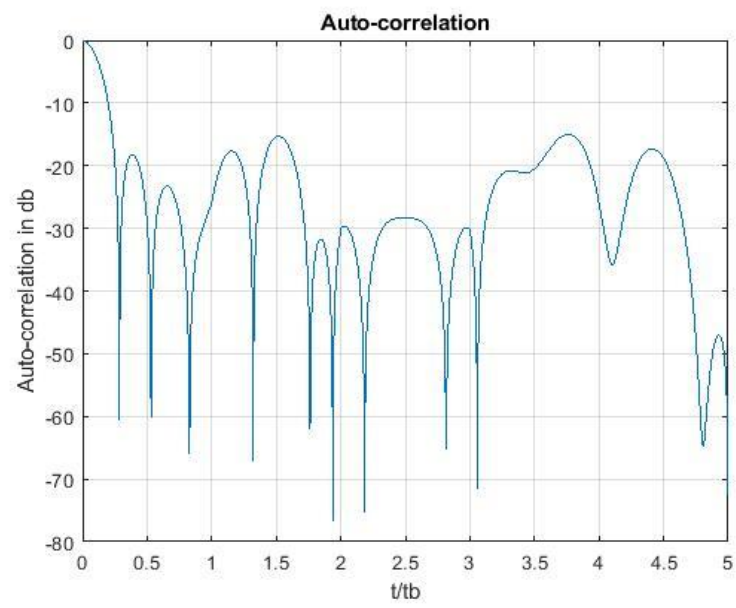

Fig. 17. Autocorrelation plot of MCPC using SC for the sequence order [ [ $\left.\begin{array}{lllll}4 & 5 & 2 & 1 & 3\end{array}\right]$

In the Table 7, a comparison of PMEPR and $1^{\text {st }}$ sidelobe level for MCPC signal based on P4 and SC is obtained for various ordering. For $\left[\begin{array}{lllll}1 & 2 & 5 & 4 & 3\end{array}\right]$ ordering an improvement of 0.7075 in PMEPR and $3.28 \mathrm{~dB}$ in $1^{\text {st }}$ sidelobe level is obtained for MCPC signal based on SC sequence method. Correspondingly for [ [ $\left[\begin{array}{lllll}4 & 3 & 1 & 2 & 5\end{array}\right]$ ordering an improvement of 0.3579 in PMEPR and $8.88 \mathrm{~dB}$ in $1^{\text {st }}$ sidelobe level is obtained for MCPC based on SC sequence method.

Table 7. Comparison of PMEPR and Sidelobe Levels of MCPC using P4 with SC technique

\begin{tabular}{|c|c|l|l|l|}
\hline \multirow{2}{*}{ Sequence } & \multicolumn{2}{|l|}{ MCPC with P4 } & \multicolumn{2}{l|}{ MCPC with SC } \\
\cline { 2 - 5 } & \multirow{2}{*}{ PMEPR } & $\begin{array}{l}\text { 1st } \\
\text { SLL }\end{array}$ & $\begin{array}{l}\text { PMEP } \\
\text { R }\end{array}$ & $\begin{array}{l}\text { 1st } \\
\text { SLL }\end{array}$ \\
\hline 13425 & 3.4821 & -13.82 & 3.6913 & -15.4 \\
\hline 35214 & 2.9764 & -13.85 & 2.6185 & -21.5 \\
\hline 45321 & 4.3988 & -13.57 & 3.6943 & -17.58 \\
\hline 32451 & 4.3988 & -11.68 & 3.6943 & -14.16 \\
\hline 45213 & 2.9764 & -14.78 & 2.6185 & -18.26 \\
\hline 34521 & 4.3988 & -13.77 & 3.5478 & -15.73 \\
\hline 43512 & 4.3988 & -11.97 & 3.6943 & -16.24 \\
\hline 43125 & 2.9764 & -14.97 & 2.6185 & -23.85 \\
\hline 12543 & 4.3988 & -12.13 & 3.6913 & -15.41 \\
\hline 34521 & 4.3988 & -13.77 & 3.5478 & -15.81 \\
\hline
\end{tabular}

Fig.18 shows comparison of autocorrelation plot of MCPC signals based on P4 and SC methods for

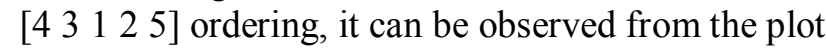
an improvement of $3.48 \mathrm{~dB}$ in 1 st sidelobe level of MCPC signal based on SC method.

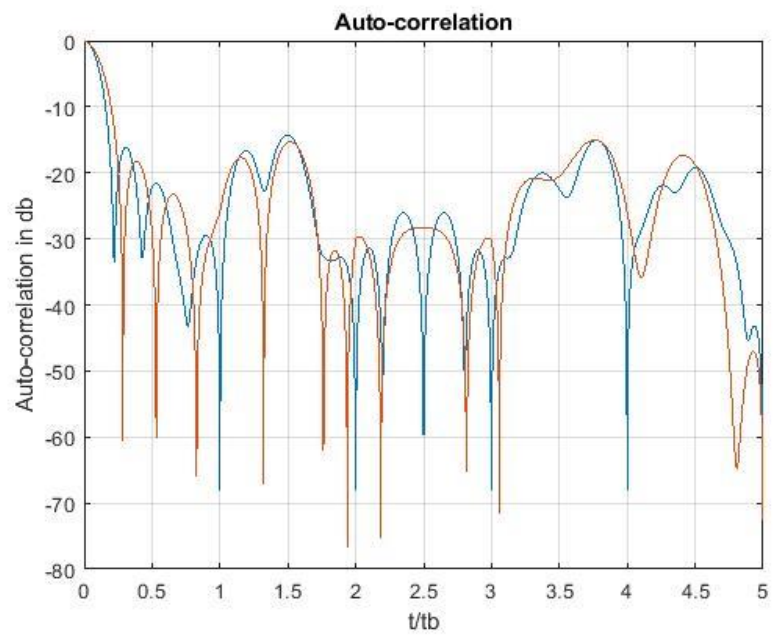

Fig. 18. Autocorrelation plots of MCPC using both $\mathrm{P} 4$ and SC technique

\section{Conclusion}

In this literature an endeavor is made to optimize the MCPC radar signal performance based on ZC and SU method. Two methods, namely ZC sequence and $\mathrm{SU}$ methods has been considered for reducing the PMEPR and sidelobe level simultaneously. An augment of $6 \mathrm{~dB}$ in $1^{\text {st }}$ sidelobe and $3 \mathrm{~dB}$ of peak sidelobe for MCPC based on ZC is attained when correlated with conventional P4 method, also an improvement of $1.36 \mathrm{~dB}$ in ISLR and $2.38 \mathrm{~dB}$ in PSLR is obtained respectively. MCPC signal based on SC approach were also developed. Numerical results in the simulation shows, for $\left[\begin{array}{lllll}1 & 2 & 5 & 4 & 3\end{array}\right]$ ordering an improvement of 0.7075 in PMEPR and $3.28 \mathrm{~dB}$ in $1^{\text {st }}$ sidelobe level is obtained for MCPC signal based on ZC sequence method. Similarly for another sequence ordering $\left[\begin{array}{lllll}4 & 3 & 1 & 2 & 5\end{array}\right]$ an improvement of 0.3579 in PMEPR and $8.88 \mathrm{~dB}$ in $1^{\text {st }}$ sidelobe level is obtained.

\section{References:}

[1] N. Levanon and E. Mozeson, "Radar Signals", John Wiley, 2004.

[2] T. Hwang, C. Yang, G. Wu, S. Li and G. Y. Li, "OFDM and its wireless applications: A survey," IEEE Trans. Veh. Technol., Vol. 58, No. 4, 2009, pp. 1673-1694.

[3] N. Levanon, "Multifrequency complementary phase-coded radar signal", IEEE Proc. Radar Sonar and Navigation, Vol. 147, No. 6, 2000, pp. 276-284. 
[4] H. Li, T. Jiang and Y. Zhou, "An improved tone reservation scheme with fast convergence for PAPR reduction in OFDM systems," IEEE Trans. Broadcast. Vol. 57, No. 4, 2011, pp. 902-906.

[5] C. Ni, Y. Ma, and T. Jiang, "A novel adaptive tone reservation scheme for PAPR reduction in large scale multi-user MIMO-OFDM systems," IEEE Wireless Commun. Letters, Vol. 5, No. 5, 2016, pp. 480-483.

[6] Jingqi Wang, Xin Lv, and Wen Wu, "SCRbased tone reservation schemes with fast convergence for PAPR reduction in OFDM system," IEEE Trans. on Information Theory, Vol. 8, No.2, 2019, pp. 624-627.

[7] S. Kiambi, E. Mwangi, G. Kamucha, "An iterative re-weighted least-squares tone reservation method for PAPR reduction in OFDM systems," WSEAS Transactions on Communications, Volume 18, 2019, pp. 153161,

[8] E. Mozeson and N. Levanon, "Multicarrier radar signals with low peak-to-mean envelope power ratio," IEE, Radar Sonar and Navigation, Vol.150, No.2, 2003, pp. 71-77.

[9] T. Huang and T. Zhao, "Low PMEPR OFDM radar waveform design using the Iterative Least Squares Algorithm," IEEE Signal Proc. Letters, Vol 22, No.11, 2015, pp. 1975-1979.

[10] G. Lellouch, A.K Mishra and M Inggs, "Design of OFDM radar rulses using genetic algorithm based techniques", IEEE Transactions on AES, Vol 52, No. 4, 2016, pp 1953-1965.

[11] C.G. Raghavendra, Sriranga R, Siddharth R Rao, Sanath M Nadig, and N.N.S.S.R.K Prasad, "A Novel approach to reduce the PMEPR of MCPC signal using random phase algorithm," Informacije MIDEM, Journal of Microelectronics, Electronics Components and Materials, Vol. 48, No. 1, 2018, pp. 63-70.

[12] D. Li, X. Dai, and H. Zhang, "Sidelobe suppression in NC-OFDM systems using constellation adjustment," IEEE Communications Letters, Vol. 13, No.5, 2009, pp. 327-329.

[13] H. Khan and S. So Joo, "Active interference restriction in OFDM-based cognitive radio network using genetic algorithm," International conference on information and communication technology convergence (ICTC), NY, 2015, pp 840-842.

[14] C. Ni, T. Jiang, and W. Peng, "Joint PAPR reduction sidelobe suppression using signal cancellation in NC-OFDM based cognitive radio systems," IEEE Trans. Veh. Technol., Vol. 64, No. 3, 2015, pp. 964-972.

[15] A. Tom, A. Sahin and H. Arslan, "Suppressing Alignment: Joint PAPR and out-of-band power leakage reduction for OFDM based systems," IEEE Trans. on Commun. Vol. 64, No. 3, 2016, pp. 1100-1109.

[16] N. A.. Sivadas and Sameer S. M, "Joint technique for sidelobe suppression and peak-toaverage power ratio reduction in noncontiguous OFDM-based cognitive radio networks," IJE, Taylor and Francis, Vol. 104, No. 2, 2016, pp. 190-203.

[17] C.G. Raghavendra, Harsha N and N.N.S.S.R.K Prasad, "Sidelobe reduction of Multicarrier Radar signals using Zad-off Chu Polyphase sequence," IEEE, International Conference on IRC applications (ICIRA 2018), 2018, pp. 1057-1061. 